\title{
Importance of cone beam computed tomography for diagnosis of calcifying cystic odontogenic tumor associated to odontoma. Report of a case
}

\author{
Yonara-Maria-Freire-Soares Marques ${ }^{1}$, Tessa-de Lucena Botelho ${ }^{1}$, Flávia-Caló-de Aquino Xavier ${ }^{1}$, Andrea- \\ Leão Rangel ${ }^{2}$, Inara-Carneiro-Costa Rege ${ }^{2}$, Andrea Mantesso ${ }^{3}$
}

\author{
${ }^{1}$ DDS,MD. Oral Pathology Departament, School of dentistry, University of São Paulo \\ ${ }^{2}$ DDS, MD. Oral Radiology Departament, School of dentistry, Paulista University \\ ${ }^{3}$ DDS, PhD. Oral Pathology Departament, School of Dentistry, University of São Paulo
}

Correspondence:

Department of Oral Pathology

School of Dentistry-University of São Paulo

Av. Lineu Prestes 2227

São Paulo/SP

CEP 05508-9000 Brazil

yonarafr@usp.br

Received: $12 / 03 / 2009$

Accepted: 12/09/2009

\author{
Marques YF, Botelho TL, Xavier FC, Rangel AL, Rege IC, Mantesso A. \\ Importance of cone beam computed tomography for diagnosis of calcifying \\ cystic odontogenic tumor associated to odontoma. Report of a case. Med \\ Oral Patol Oral Cir Bucal.2010 May 1;15 (3):e490-3. \\ http://www.medicinaoral.com/medoralfree01/v15i3/medoralv15i3p490.pdf \\ Article Number: 2744 http://www.medicinaoral.com/ \\ (C) Medicina Oral S. L. C.I.F. B 96689336 - pISSN $1698-4447$ - eISSN: 1698-6946 \\ eMail: medicina@medicinaoral.com \\ Indexed in: \\ -SCI EXPANDED \\ -JOURNAL CITATION REPORTS \\ -Index Medicus / MEDLINE / PubMed \\ -EMBASE, Excerpta Medica \\ -SCOPUS \\ -Indice Médico Español
}

\begin{abstract}
The calcifying cystic odontogenic tumour (CCOT) is a rare benign cystic neoplasm not infrequently associated with odontoma. This report documents a case of CCOT associated with compound odontoma arising in the anterior maxilla in a 25 -year-old woman. Conventional radiographs showed a large calcified mass with poorly visualized radiolucent margins. The extent and condition of the internal structure of the CCOT associated with odontoma was able to be determined based on radiographic findings from cone beam computed tomography. This advanced image technique proved to be extremely useful in the radiographic assessment of this particular neoplasm of the jawbones.
\end{abstract}

Key words: Cone beam computed tomography, conventional radiographs, calcifying cystic odontogenic tumour, compound odontoma.

\section{Introduction}

The calcifying odontogenic cyst (COC) is a benign cystic neoplasm of odontogenic origin that was first described as a distinct entity by Ledesma-Montes et al. (1) in 1962 and has recently been defined as a calcify ing cystic odontogenic tumour (CCOT) by the World Health Organization (WHO) as a result of its neoplastic behavior. CCOT is characterized by an ameloblastoma- like epithelium with ghost cells that may calcify (WHO) (1).

The CCOT may occur in association with other odontogenic tumours, most commonly the odontoma, occurring in about $24 \%$ of cases (2). The authors relate a case of CT used as an auxiliary method for diagnosis and the treatment of a calcifying odontogenic cyst associated with odontoma. 


\section{Case Report}

A 25-year-old woman was referred by her endodontist for management of a mixed radiolucent and radiopaque lesion located in the anterior maxilla, detected during routine radiographic examination. A clinical examination revealed growth in alveolar process near to the apex of the upper right central incisor with a normal mucosal aspect (Fig. 1A). Only a discrete palatal swell ing in the upper right central incisor area was seen. Intraoral radiographs showed a unilocular radiopaque image consisting of a central coalescent area and many structures with resemblance to small teeth in the adjacent region (Fig. 1B). These radiopaque images were well circumscribed by a large radiolucent halo, overlapping the root of the central incisor. A presumptive diagnosis of compound odontoma was made. However, an irregular radiolucent area, associated with the radiopaque lesion in the superior limit was seen. The presence of this image, together with the discrete clinical swelling, uncommon in odontoma, led the dentist to request cone beam computed tomographic images, which revealed large high-density masses at the palatine margin, connected to a well-defined expanding lesion with a thinned cortical vestibular plate (Fig. 1C and D). A thick cystic wall and mineralized material, compatible with an odontoma, was removed by surgical enucleation under local anaesthesia and a palatal surgical approach (Fig. 2A). Microscopically, the cystic wall was lined by an ameloblastomatous epithelium with numerous ghost cells and calcified particles (Fig. 2B and C). There was a mixture of dental hard tissues comprised of dentin, enamel matrix, cementum and connective tissue (Fig. 2D). The histopathologic diagnosis of a calcifying odontogenic cyst associated with compound odontoma was made. After surgical treatment, no recurrence was found after 8 months of follow-up.

\section{Discussion}

The CCOT is a new designation for calcifying odontogenic cyst (COC) that was reclassified in 2005 by the current World Health Organization (WHO) classification (1). The CCOT is clinically characterized as
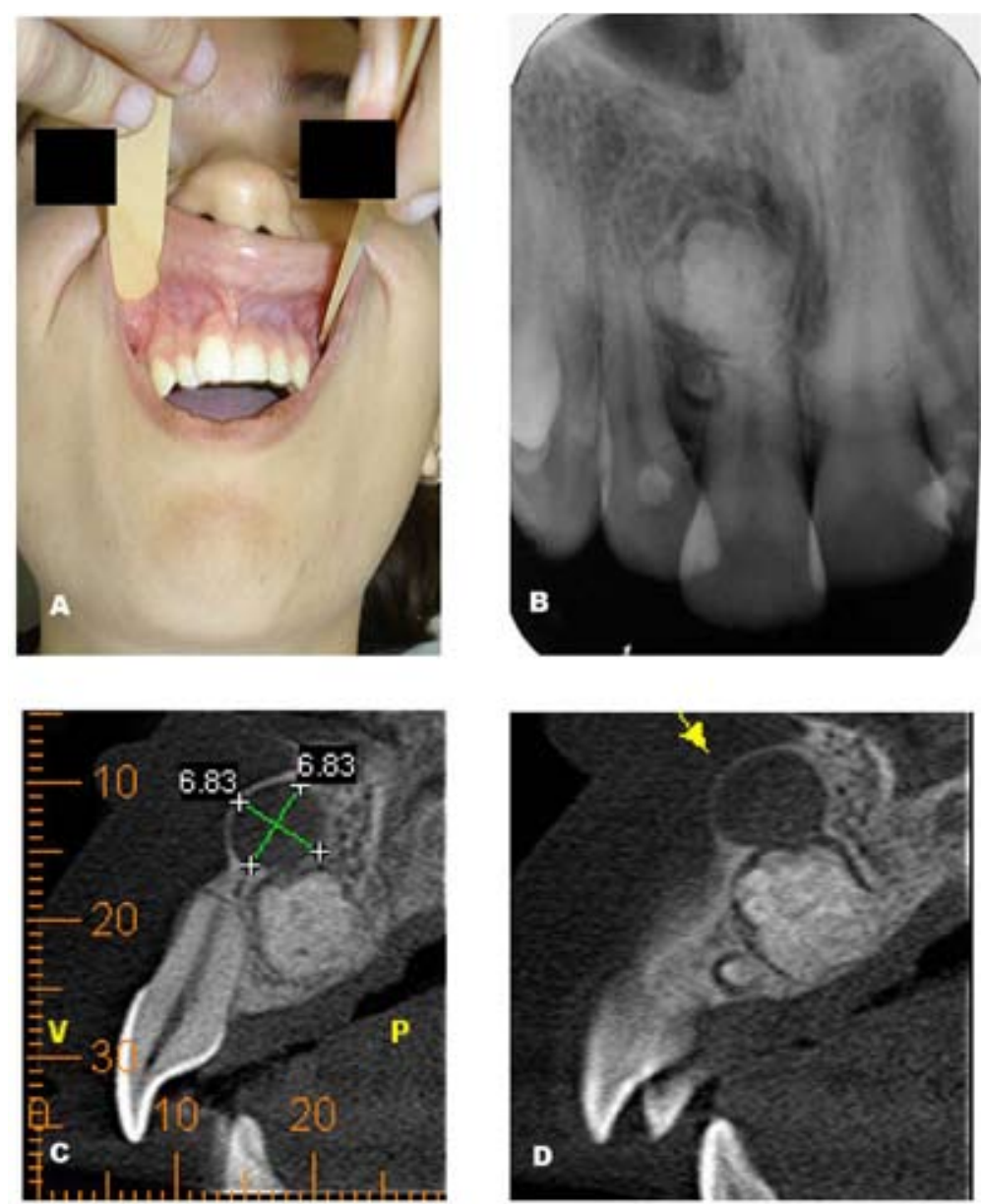

Fig. 1A. Clinical aspect - growth in alveolar process near to the apex of the upper right central incisor; B) Periapical radiograph - radiopaque image circunscribed by a large radiolucent halo overlaps the root of the central incisor; C) and D) Cone beam computed tomography - large high-density masses at the palatine margin, connected to a well-defined expanding lesion with a thinned cortical vestibular plate. 

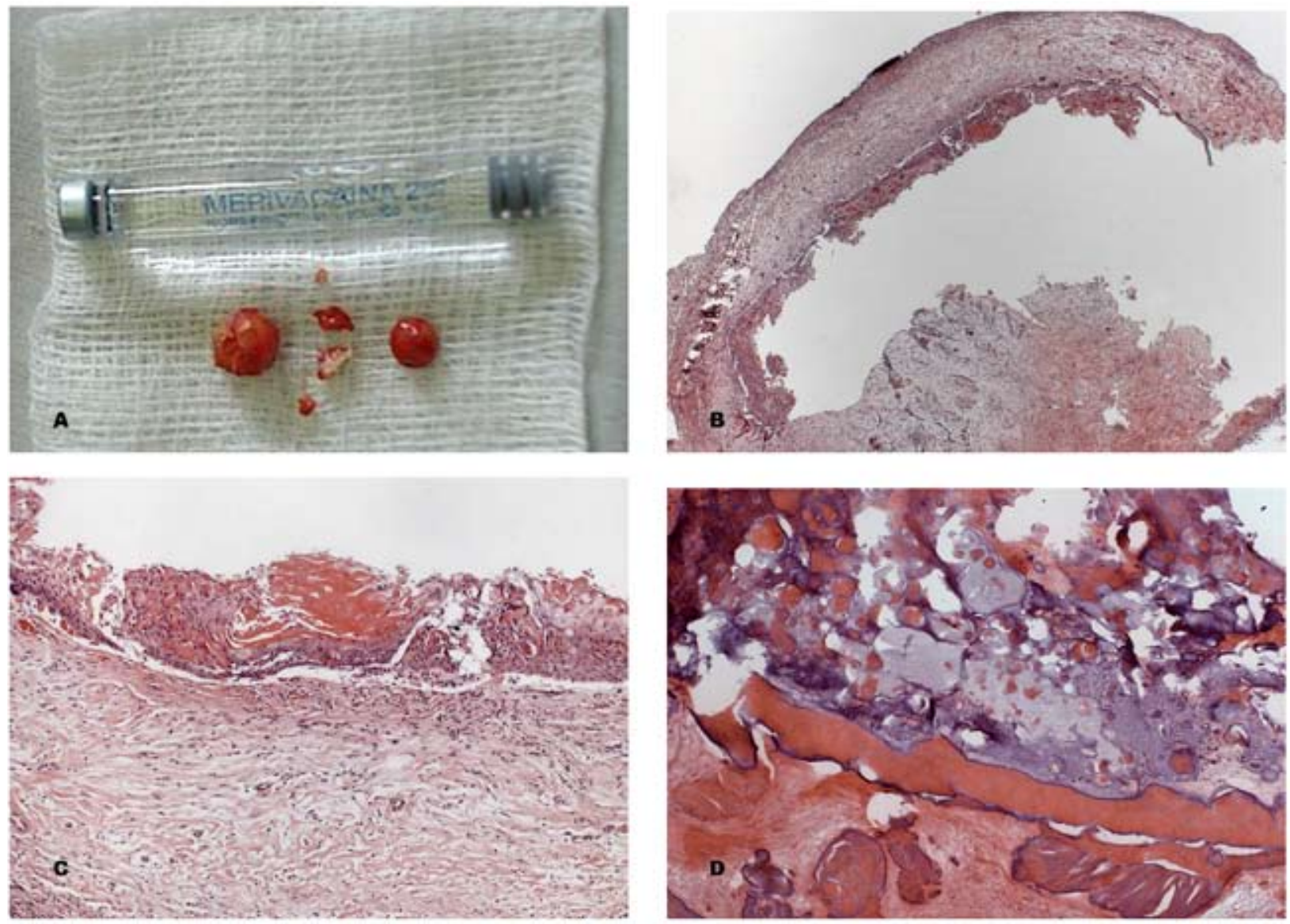

Fig. 2A. Macroscopical aspect of fragments of structures resembling small teeth; B) Microscopical findings of the cystic wall and fibrous capsule; C) High power view of cystic wall with ameloblastomatous epithelium containing numerous ghost cells; D) Microscopical image of mixture of dental hard tissues.

a painless slow-growing tumor, that equally affects the maxilla and mandible, with predilection for the anterior region of the jaw $(2,3)$. It has a peak of incidence in the second and third decades, with a mean age of 30.3 years and without any gender predilection $(2,4)$. The treatment of choice for the CCOT is conservative surgical enucleation. Recurrences are very uncommon but a long follow-up period seems advisable $(2,5)$.

Odontogenic tumors of the jaw bones show a wide spectrum of radiographic manifestations resulting from the fact that the jaw bones contain dental components of ectodermal and mesodermal origin. Specifically, since the CCOT is a mixed lesion, it has a wide range of radiologic appearances, mimicking a number of different pathological conditions. Radiographically, it may appears as an unilocular or multilocular radiolucent area with either well circumscribed or poorly defined margins that also may be observed in association with an unerupted tooth (6). Calcification is an important radiographic feature in the interpretation of CCOT, but it is detected in only about half of the reported cases. CCOT may be evident in the lumen of the cystic image; however, when it is present in small amounts it may not be recognized radiologically (7).

Additionally, the CCOT is frequently found in association with various types of odontogenic tumors, such as complex/compound odontomas, ameloblastomas, and ameloblastic fibromas (WHO), but the most common of them is the odontoma, occurring in about $24 \%$ of cases $(1,2)$. However, this association is a challenge for diagnosis using conventional images, due to presence of numerous accidents in anatomical structures of the jaw region. In the present case, an intraoral X-ray was unable to reveal useful information for the correct diagnosis of the lesion, since overlapped images could only reveal a unilocular radiopaque image with multiple structures that resembled small teeth (Figure 1B), leading only to a presumptive diagnosis of odontoma.

Recently, CT examinations have been applied to this kind of lesion with benefits and features being emphasized in many case reports (8). Some reports have indicated that this technique may be more useful than plain film radiography to confirm the presence of calcifications along the cyst wall, which may not be detected 
by simple radiographs due to the superimposition of anatomic details. CT examination for CCOT diagnosis is also capable of detecting increased attenuation areas due to desquamated keratin, another histological feature of COC (7).

There is no previous report showing the value of conebeam tomography in CCOT. Cone-beam CT (CBCT) is also known as cone-beam volumetric tomography and CBCT machines have two major differences compared with so-called "medical" CT scanners. First, CBCT uses a low-energy fixed anode tube, similar to that used in dental panoramic x-ray machines. Secondly, CBCT machines rotate around the patient only once, capturing the data using a cone-shaped x-ray beam. These changes allow a less expensive, smaller machine that exposes the patient to approximately $20 \%$ of the radiation of an helical CT, equivalent to the exposure from a full-mouth periapical series. Due to a difference in voxel size, CBCT of a limited area is also very effective in achieving high spatial resolution in comparison with conventional CT (9).

Clinically, CBCT has a wide range of applications; for example, it may be used to determine the extent and condition of the internal structure of lesions, the fracture of teeth roots, the condition of impacted teeth and pathologic conditions in jaw bones (8). Besides, the benefits for diagnostic processes, three-dimensional imaging of cysts and tumors of the maxillofacial region can provide the surgeon with vital information necessary for planning surgery (10).

In the present case, we emphasize that the CBCT image allowed a tridimensional visualization that, besides showing the association between lesions, revealed an unusual presentation of this lesion, since the cystic cavity and odontoma could be viewed separately. It was only possible to identify the cystic lesion and to plan the treatment properly after the use of CBCT images.

In conclusion, even though the conventional X-ray images were not capable of identifying the cystic lesion, a CBCT was required to clarify detailed structures of a limited area, demonstrating the margins of the connected lesions, as well as their internal architecture. In addition, it could be shown by this case report that the CBCT images can achieve high spatial resolution in comparison with conventional X-rays, reducing the radiation dose and also guiding the treatment of this intraosseous lesion by establishing the relationship between adjacent structures. Finally, CBCT proved to be extremely useful for diagnostic purposes of this neoplasm in jawbones, which were unclear in the bidimentional images. Therefore, details of the radiographic pattern and the internal structures visualized by CBCT images served as an important tool in reaching an adequate diagnosis.

\section{References}

1. Ledesma-Montes C, Gorlin RJ, Shear M, Prae Torius F, MosquedaTaylor A, Altini M, et al. International collaborative study on ghost cell odontogenic tumours: calcifying cystic odontogenic tumour, dentinogenic ghost cell tumour and ghost cell odontogenic carcinoma. J Oral Pathol Med. 2008;37:302-8.

2. Buchner A. The central (intraosseous) calcifying odontogenic cyst: an analysis of 215 cases. J Oral Maxillofac Surg. 1991;49:330-9.

3. Hirshberg A, Kaplan I, Buchner A. Calcifying odontogenic cyst associated with odontoma: a possible separate entity (odontocalcifying odontogenic cyst). J Oral Maxillofac Surg. 1994;52:555-8.

4. Hong SP, Ellis GL, Hartman KS. Calcifying odontogenic cyst. A review of ninety-two cases with reevaluation of their nature as cysts or neoplasms, the nature of ghost cells, and subclassification. Oral Surg Oral Med Oral Pathol. 1991;72:56-64.

5. Johnson A 3rd, Fletcher M, Gold L, Chen SY. Calcifying odontogenic cyst: a clinicopathologic study of 57 cases with immunohistochemical evaluation for cytokeratin. J Oral Maxillofac Surg. 1997;55:679-83.

6. Devlin H, Horner K. The radiological features of calcifying odontogenic cyst. Br J Radiol. 1993;66:403-7.

7. Iida S, Fukuda Y, Ueda T, Aikawa T, Arizpe JE, Okura M. Calcifying odontogenic cyst: radiologic findings in 11 cases. Oral Surg Oral Med Oral Pathol Oral Radiol Endod. 2006;101:356-62.

8. Araki M, Kameoka S, Mastumoto N, Komiyama K. Usefulness of cone beam computed tomography for odontogenic myxoma. Dentomaxillofac Radiol. 2007;36:423-7.

9. Hashimoto K, Kawashima S, Araki M, Iwai K, Sawada K, Akiyama Y. Comparison of image performance between cone-beam computed tomography for dental use and four-row multidetector helical CT. J Oral Sci. 2006;48:27-34.

10. Quereshy FA, Savell TA, Palomo JM. Applications of cone beam computed tomography in the practice of oral and maxillofacial surgery. J Oral Maxillofac Surg. 2008;66:791-6. 